\title{
Circular RNAs: Functions and Clinical Significance in Cardiovascular Disease
}

\author{
Lei Zhang ${ }^{1 *}$, Yuan Zhang ${ }^{1}$, Yin Wang ${ }^{1}$, Yanfang Zhao', Han Ding ${ }^{1}$ and Peifeng Li ${ }^{1 *}$ \\ ${ }^{1}$ Institute for Translational Medicine, The Affiliated Hospital of Qingdao University, Qingdao University, Qingdao, China, \\ ${ }^{2}$ Institute of Biomedical Research, School for Life Science, Shandong University of Technology, Zibo, China
}

OPEN ACCESS

Edited by:

Rebecca Ann Wingert,

University of Notre Dame,

United States

Reviewed by:

Myriam Gorospe,

National Institutes of Health $(\mathrm{N} / \mathrm{H})$,

United States

Bin Liu,

Mississippi State University,

United States

Dachun Xu,

Tongji University, China

*Correspondence:

Lei Zhang

leizhang@qdu.edu.cn

Peifeng Li

peifli@qdu.edu.cn

Specialty section:

This article was submitted to

Molecular Medicine,

a section of the journal

Frontiers in Cell and Developmental

Biology

Received: 16 July 2020 Accepted: 09 September 2020 Published: 29 September 2020

Citation:

Zhang $L$, Zhang $Y$, Wang $Y$,

Zhao Y, Ding $H$ and Li P (2020) Circular RNAs: Functions and Clinical

Significance in Cardiovascular

Disease.

Front. Cell Dev. Biol. 8:584051. doi: 10.3389/fcell.2020.584051
Cardiovascular disease (CVD) causes high morbidity and mortality worldwide. Accumulating research has indicated the possible roles played by circular RNAs (circRNAs) in the pathogenesis of CVD. CircRNAs are non-coding RNAs with covalently closed loop structures. CircRNAs can function by acting as miRNA sponges, RNA binding protein sponges, mRNA transcriptional regulators and templates for protein translation. The specific characteristics of circRNAs such as high stability, abundant distribution, and tissue- and developmental stage-specific expression make them potential biomarkers for the diagnosis and prognosis of CVD. In this paper, we systematically summarized the current knowledge regarding the biogenesis, biological properties and the action mechanisms of circRNAs, elucidated the roles played by circRNAs in the pathogenesis of CVD, and explored the diagnostic potential of circRNAs in CVD. With in-depth studies, an increasing number of molecular mechanisms underlying the participation of circRNAs in CVD may be elucidated, and the application of circRNAs in the clinical diagnosis and prevention of CVD may eventually be realized.

Keywords: cardiovascular disease, circular RNAs, pathogenesis, diagnosis, clinical application

\section{INTRODUCTION}

Cardiovascular disease (CVD) is one of the leading causes of morbidity and mortality worldwide. In recent decades, scientists have made considerable progress in the diagnosis and treatment of CVD. However, the increasing tendency of the mortality rate of CVD has not been stopped to date. Therefore, novel and effective strategies for the diagnostic and therapeutic interventions of CVD are strongly warranted. A growing number of studies have determined that non-coding RNAs, such as microRNAs (miRNAs) and long non-coding RNAs (lncRNAs), participate in the pathological processes of CVD and can serve as biological markers in diagnosis, prognosis and clinical treatment (Yang et al., 2016; Zhang L. et al., 2018, 2020). In the last several years, circular RNAs (circRNAs) have also been reported to be associated with CVD.

CircRNAs are covalently closed loop structures with no $5^{\prime}$ cap and $3^{\prime}$ polyadenylated tail. CircRNAs were first identified in plant viruses (Kolakofsky, 1976) and were thought to have no function or very limited function (Nigro et al., 1991; Cocquerelle et al., 1992; Capel et al., 1993). Subsequently, the existence of circRNAs has also been reported in many organisms, such as yeast (Schroeder et al., 1983) and humans (Cocquerelle et al., 1993). The rapid development of prediction, detection and screening technologies for circRNAs facilitates the discovery of different types of circRNAs. Studies have reported that circRNAs might participate in the regulation of physiological and pathological processes of different kinds of CVD (Fan et al., 2017; Lim et al., 2020), such as myocardial infarction (MI) (Geng et al., 2016; Cai et al., 2019), cardiac senescence 
(Du et al., 2016; Chen et al., 2018) and coronary artery disease (CAD) (Holdt et al., 2016; Dang et al., 2017; Shan et al., 2017). CircRNAs have a variety of characteristics, including high stability, tissue-and developmental-specific expression, and the altered expression in the pathological and normal conditions of various diseases (Werfel et al., 2016; Siede et al., 2017; Gupta et al., 2018). Due to these characteristics, circRNAs exhibit considerable potential as biomarkers for the detection of CVD from human blood samples (Vausort et al., 2016; Zhao et al., 2017). In this paper, we will summarize the available knowledge on the biogenesis of circRNAs, the functions of circRNAs, the roles of circRNAs in CVD and the diagnostic potential of circRNAs in CVD.

\section{BIOGENESIS OF CircRNAS}

CircRNAs are divided into three categories: exonic circRNAs (ecircRNAs or ecRNAs) (Zhang et al., 2014), circular intronic RNAs (ciRNAs) (Zhang et al., 2013) and exon-intron circRNAs (EIciRNAs) (Li et al., 2015). CircRNAs are generated from premRNAs through backsplicing. Four mechanisms of circRNA formation have been revealed. The $5^{\prime}$ end of the intron (splice donor site, GU) and the $3^{\prime}$ end of the intron (splice acceptor site, AG) can be covalently bound to generate an exon-containing lariat, which will be internally spliced thereafter to form an exonic circle (Jeck et al., 2013; Jeck and Sharpless, 2014) (Figure 1A). The RNA base motifs (e.g., Alu repeats) in the introns of pre-mRNA can pair with the complementary sequences (Jeck et al., 2013; Zhang et al., 2014), and direct cyclization subsequently occurs to generate ecRNAs (introns removed) or EIciRNAs (introns retained) (Jeck et al., 2013) (Figure 1B). In the introns, the C-rich element close to the branch and the GU-rich element close to the $5^{\prime}$ splice site can bind together, and then the other exons and introns are removed by the spliceosome to form ciRNAs (Zhang et al., 2013) (Figure 1C). The bridging of RNA binding proteins (RBPs) with pre-mRNAs has also been elaborated to facilitate the production of circRNAs (ecRNAs or EIciRNAs) (Ashwal-Fluss et al., 2014; Conn et al., 2015) (Figure 1D).

Multiple factors participate in the biogenesis of circRNAs. Quaking (QKI) and Muscleblind (MBL) proteins function as regulatory activators of the biogenesis of circRNAs (AshwalFluss et al., 2014; Conn et al., 2015). In contrast, an adenosine deaminase acting on RNA-1 inhibits the circRNA formation by destroying the stem structures (Rybak-Wolf et al., 2015). Serine-arginine and heterogeneous nuclear ribonucleoprotein can regulate the generation of circRNAs in Drosophila (Kramer et al., 2015).

\section{BIOLOGICAL PROPERTIES OF CircRNAS}

CircRNAs have some common characteristics and the most important ones are elaborated as follows.

(1) Wide distribution and diversity. CircRNAs are found in many eukaryotic organisms ranging from plants to animals and in all tissues (Jeck et al., 2013). In humans, over 30,000
circRNAs have been found and the number will increase in the future (Xu et al., 2017; Zeng X. X. et al., 2017).

(2) High stability. Because of the covalently closed structures, circRNAs are resistant to the degradation by ribonuclease (RNase) and are more stable than linear RNAs (Suzuki et al., 2006).

(3) Specific expression. CircRNAs are specifically expressed in different tissues, cells and developmental stages (Jakobi et al., 2016; Li Y. S. et al., 2017; Xu et al., 2017). circRNAs have different profiles at four stages of heart differentiation (Li Y. S. et al., 2017). Significant alterations have been detected at different developmental stages in cardiomyocytes derived from induced pluripotent stem cells (Siede et al., 2017).

(4) Evolutionary conservation. Many circRNAs seem to be evolutionarily conserved among species (Jeck et al., 2013; AbouHaidar et al., 2014). Jeck et al. (2013) found the homology of 2121 circRNAs between human fibroblasts and mouse genome. Werfel et al. (2016) reported high homology of 1288 circRNAs across human, mouse and rat. However, Werfel et al. (2016) also revealed that only a small number of circRNAs were conserved. Other studies have also illustrated that many circRNAs are specific to species (Aufiero et al., 2019; Lim et al., 2020).

(5) Dynamic expression profiles between normal and pathological conditions. A lot of circRNAs have altered expression related to diseases. Zheng et al. (2016) revealed altered expression of many circRNAs between normal tissues and cancerous tissues. In many other diseases, the expression differences of circRNAs have also been verified (Werfel et al., 2016; Siede et al., 2017; Gupta et al., 2018). Some studies reported lack of dynamic expression of circRNAs in specific diseases (Werfel et al., 2016; Tan et al., 2017).

\section{THE UNDERLYING MECHANISMS OF CircRNA FUNCTIONS}

The characteristics of circRNAs, such as wide distribution, high stability, expression specificity and localization, indicate that circRNAs have various biological functions. Recent studies have illustrated that circRNAs can function through different mechanisms (Figure 2).

\section{Some CircRNAs Act as miRNA Sponges}

CircRNAs contain miRNA response elements (MREs) that facilitate the binding of circRNAs and miRNAs. This binding decreases the level of functional miRNAs and thus increases the expression of miRNA targets (Hansen et al., 2013a; Tay et al., 2014). This process is known as the "sponge effect," as circRNAs can absorb miRNAs similar to sponges (Figure 2A). Many circRNAs can function as miRNA sponges. CiRS-7/CDR $1_{\text {as }}$ contains more than 70 binding sites for miR-7 that are involved in the pathogenesis of various diseases (Hansen et al., 2013b; Pan et al., 2018; Liu et al., 2019). The binding of ciRS-7/CDR1as to miR-7 causes downregulation of miR-7 and elevated levels 


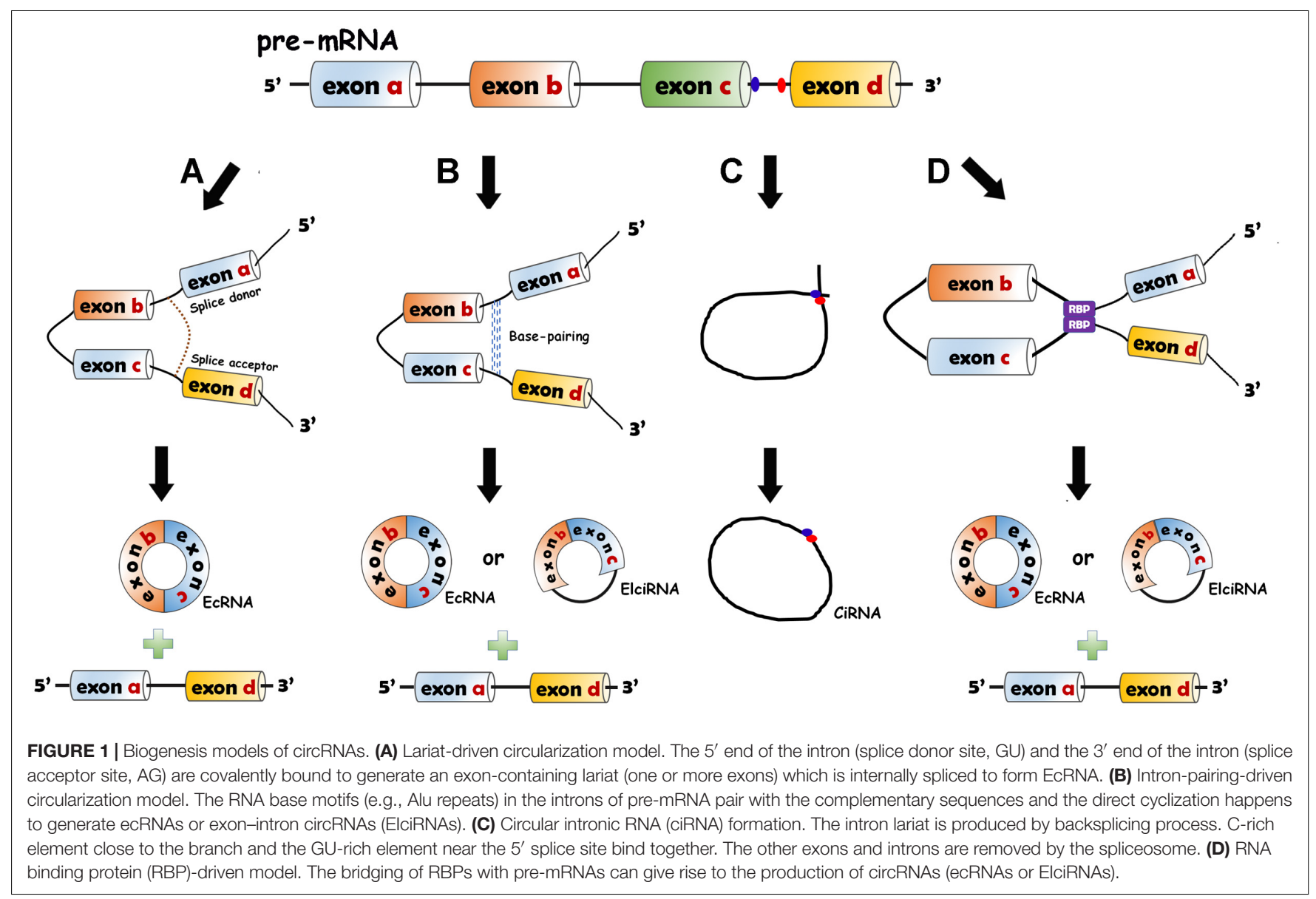

of miR-7 target genes. Sry, a testis-specific circRNA, has 16 conserved MREs for miR-138 (Hansen et al., 2013a). The activity of miR-138 is inhibited due to the sponge effect and the miR-138 target genes are upregulated (Hansen et al., 2013a). CircHIPK3 have different MREs (total number: 18) which allow it to bind to nine different miRNAs (Zheng et al., 2016). HRCR can bind to miR-223 which promotes the pathogenesis of cardiac hypertrophy and heart failure (HF) (Wang et al., 2016). CircNFIB can competitively absorb miR-433 to enhance cardiac fibroblast proliferation induced by the stimulation of TGF- $\beta$ (Zhu et al., 2019). CircRNA_000203 can directly sponge miR-26b-5p and miR-140-3p to regulate the occurrence of cardiac hypertrophy (Li et al., 2019).

\section{Some CircRNAs Serve as RBP Sponges}

CircRNAs can interact with RBPs and inhibit RBP activity (Figure 2B). CircMbl is produced from the same pre-mRNA as the $\mathrm{MBL}$ protein and can absorb $\mathrm{MBL}$ proteins to regulate the subsequent physiological processes (Ashwal-Fluss et al., 2014). CircPABPN1 can bind to HuR, which is a well-known RBP, to prevent the interaction between HuR and PABPN1 mRNA, suppressing the translation of PABPN1 mRNA (Abdelmohsen et al., 2017). CircANRIL competitively recruits PES1 (an essential 60S-preribosomal assembly factor), leading to inhibition of ribosome biogenesis (Holdt et al., 2016). CircFoxo3 participates in the processes of cardiomyocyte senescence and cell cycle progression through interacting with different RBPs, such as anti-senescent protein ID-1, transcription factor E2F1, anti-stress proteins FAK, p21 (cyclin-dependent kinase inhibitor 1) and CDK2 (cyclin-dependent kinase 2) (Du et al., 2017). CircAmotl1 can protect cardiomyocytes and promote cell proliferation and wound repair by binding to the cardioprotective molecules (PDK1 and AKT1) and to STAT3 (signal transducer and activator of transcription 3) (Yang Z. G. et al., 2017; Zeng Y. et al., 2017).

\section{Some CircRNAs Encode Peptides}

Studies conducted in recent years have demonstrated that circRNAs can serve as templates for protein translation (Chen and Sarnow, 1995; Wesselhoeft et al., 2018) (Figure 2C). It was first observed in prokaryotes that circRNAs were able to encode proteins. In 1986, studies in hepatitis D virus showed that circRNAs could be translated into functional proteins (Kos et al., 1986). In Escherichia coli, a circRNA was also reported to act as a translation template (Perriman and Ares, 1998). CircRNAs lack a $5^{\prime}$ cap and a $3^{\prime}$ polyadenylated tail which are typical translation initiation structures of linear RNAs. Nevertheless, instead of recruiting ribosomes, circRNA translation is initiated with the help of specific elements, such as IRES (internal ribosome entry 


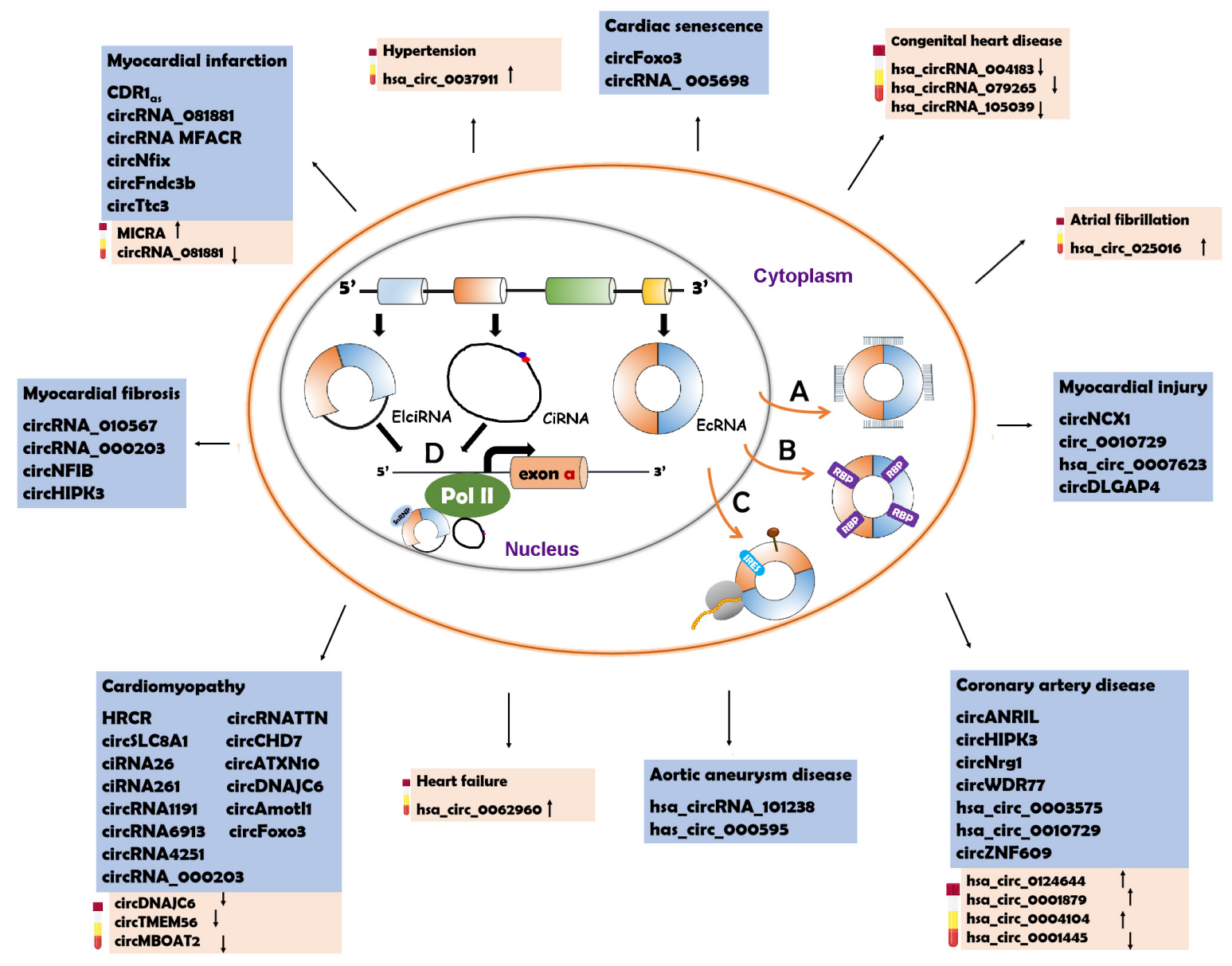

FIGURE 2 | Underlying action mechanisms of circRNAs and the circRNAs relevant to CVD pathogenesis. (A) CircRNAs can serve as miRNA sponges to regulate the functions of miRNAs. (B) CircRNAs can bind to RNA binding proteins (RBPsש) to influence their functions. (C) CircRNAs can be the templates to encode proteins with the help of internal ribosome entry site (IRES sis) elements or $\mathrm{m}^{6} \mathrm{~A}$-modification (brown pin ${ }^{\mathbf{T}}$ ). (D) circRNAs with intronic sequences can regulate the expression of parental genes through binding to RNA polymerase II (Pol II). The circRNAs involved in CVD are listed in the blue box. The possible diagnostic biomarkers are displayed in the pink box. EcRNA, exonic circRNA; ElciRNA, exon-intron circRNA; CiRNA, circular intronic RNA.

site) and $N$-methyladenosine $\left(\mathrm{m}^{6} \mathrm{~A}\right)$ (Chen and Sarnow, 1995; Wesselhoeft et al., 2018). When IRESs are introduced into a circRNA, the synthetic circRNA initiates translation (Chen and Sarnow, 1995). CircZNF609 has an IRES element and can be translated into a protein that functions in myoblast proliferation (Legnini et al., 2017). CircFBXW7 can be translated into a functional protein that plays a role in the inhibition of glioma tumorigenesis with the assistance of the IRES element (Yang et al., 2018). $\mathrm{m}^{6} \mathrm{~A}$ modification has been illustrated to facilitate the translation of linear mRNAs in a cap-independent manner (Meyer et al., 2015). Some circRNAs also employ $\mathrm{m}^{6} \mathrm{~A}$ to initiate translation (Yang Y. et al., 2017). The $\mathrm{m}^{6} \mathrm{~A}$ reader YTHDF3 can interact with circRNAs and subsequently recruit translation initiation factors to drive the initiation of circRNA translation (Yang Y. et al., 2017). In a study based on ribosome profiling of Drosophila heads, circMbl3 was demonstrated to be the template for splicing-dependent translation (Pamudurti et al., 2017). Another recent study found that $\operatorname{circ} \beta$-catenin could be translated into the $\beta$-CATENIN isoform, a functional protein that activates the $\mathrm{Wnt} / \beta$-catenin pathway and promotes tumor development in liver cancer (Liang et al., 2019).

\section{Some CircRNAs Regulate the Transcription of Parental Genes}

Of the three types of circRNAs, ecRNAs account for the majority and are predominantly located in the cytosol (Hansen et al., 2013a; Memczak et al., 2013). The cytoplasmic localization of ecRNAs facilitates their functions as miRNA sponges, RBP sponges and translational templates. CiRNAs and EIciRNAs are confined to the nucleus due to their intronic sequences (Zhang et al., 2013). Recent studies have verified the roles of nucleuslocalized ciRNA and EIciRNA in the transcriptional regulation of their parental genes (Zhang et al., 2013; Li et al., 2015) (Figure 2D). CiRNAs can directly interact with RNA polymerase II (Pol II) and enhance parental gene transcription (Zhang et al., 2013), whereas EIciRNAs bind to the U1 small nuclear ribonucleoproteins (snRNPs) at first, and the complex promotes 
the interaction with Pol II (Li et al., 2015). CiRNA-ankrd52 can bind to Pol II of the pre-mRNA of the ANKRD52 gene to promote transcription, while the knockdown of ciankrd52 may cause a significant transcriptional decrease in the parental gene (Zhang et al., 2013). CircEIF3J and circPAIP2 are EIciRNAs that can form a complex with U1 snRNP and Pol II (Li et al., 2015). In general, ciRNAs and EIciRNAs can serve as transcription regulators of their parental genes.

\section{CircRNA IDENTIFICATION AND RESEARCH DATABASES}

Various methods have been developed to identify and study the functions of circRNAs. RNA-seq, microarray, Northern Blot and RT-PCR analyses are the most widely used. RNAseq can identify new circRNA species and quantify circRNA expression. Next-generation sequencing and depletion of ribosomal RNA methods may be cooperatively utilized for circRNA-seq. The microarray method can only detect and quantify known circRNAs approximately and the results require further confirmation. For high-throughout sequencing, reliable and precise algorithms are of vital importance. Many algorithms have been developed, such as circRNA-finder, MapSplice, CIRCexplorer, and CIRI (Hansen et al., 2016). The combined use of these algorithms may improve the accuracy and sensitivity of circRNA identification. Northern Blot and RT-PCR methods only validate known circRNAs dependent on an exoribonucleaseRNase R, which cleaves linear RNAs from the $3^{\prime}$ end (Asha et al., 1983). With circular structures, circRNAs can avoid the cleavage of RNase R. Thus, in the extraction process, total RNA is digested by RNase $\mathrm{R}$ to discard the linear RNAs (Szabo and Salzman, 2016). The collected RNAs are then combined with specific probes (Northern Blot method) or reverse-transcribed to obtain cDNAs of circRNAs (RT-PCR method). Aside from these methods for circRNA identification, some other methods are employed to analyze the subcellular localization (fluorescence in situ hybridization) or the interaction between circRNAs and miRNAs/RBPs (e.g., RNA immunoprecipitation and dual luciferase reporter assay) (Li Y. et al., 2018; Zirkel and Papantonis, 2018). In addition to these known methods, simpler and more efficient methods are still urgently needed for the identification and characterization of circRNAs.

To elucidate the roles of circRNAs, many online databases have been developed (Table 1). Different databases emphasize on different aspects of circRNA research including prediction, identification, protein-coding annotation, circRNA-miRNA interactions and circRNA-RBP interactions. The combined use of these databases may help to establish a foundation for the further studies of circRNAs.

\section{CircRNAs AND CARDIOVASCULAR DISEASES}

With the use of advanced technologies in sequencing and data analysis, a lot of circRNAs have been detected in human hearts and been reported to be associated with CVD (Jakobi et al., 2016; Werfel et al., 2016; Fan et al., 2017; Tan et al., 2017) (Table 2 and Figure 2). Moreover, circRNAs have been observed to have great potential as biomarkers for the diagnosis and prognosis of CVD (Vausort et al., 2016; Sonnenschein et al., 2019; Wu et al., 2019; Vilades et al., 2020) (Table 3 and Figure 2).

\section{Myocardial Infarction}

Myocardial infarction provokes cardiac remodeling and is often complicated by arrhythmia, shock or HF. CDR $1_{a s}$ has been determined to sponge miR-7 and to have elevated levels in in mice with MI (Geng et al., 2016). The increased levels of $\mathrm{CDR} 1_{\text {as }}$ can upregulate PARP and SP1, the targets of miR-7, which may subsequently enlarge the size of MI (Geng et al., 2016). CircRNA_081881 has been found to be associated with acute MI. CircRNA_081881 has seven binding sites for miR548 , and the competitive binding upregulates the expression of PPAR $\gamma$ which can protect the heart from acute MI (Deng et al., 2016). MTP18 participates in the development of MI. CircRNA MFACR could absorb miR-652-3p to increase the expression of MTP18 and subsequently promote the progression of MI (Wang et al., 2017). CircNfix has been revealed to promote the degradation of Ybx1 and sponge miR-214. The downregulation of circNfix inhibited cardiomyocyte apoptosis after MI and promoted cardiac regeneration and repair (Huang et al., 2019). CircFndc3b exhibited significantly decreased expression levels in post-MI mouse hearts. CircFndc3b could function through interacting with RBP-FUS which regulates the expression and the signaling pathway of vascular endothelial growth factorA (VEGF-A). The overexpression of circFndc3b in post-MI mouse hearts could decrease cardiomyocyte apoptosis and fibrosis, enhance neovascularization, reduce infarct size and attenuate left ventricular dysfunction after MI (Garikipati et al., 2019). CircTtc3 was reported to be significantly upregulated in rats with MI (Cai et al., 2019). CircTtc3 could recruit miR-15b-5p to repress its inhibitory effect on Arl2, an ADP-ribosylation factor relevant to cardiomyocyte viability (Cai et al., 2019). The knockdown of circTtc3 exacerbated the symptoms of cardiac dysfunction post-MI, suggesting that circTtc3 plays a role in the cardiac protection in MI (Cai et al., 2019).

\section{Myocardial Fibrosis}

Myocardial fibrosis is a disease condition in which normal myocardium is replaced by non-beating cardiac fibroblasts, resulting in diastole difficulty. CircRNA_010567 exhibited significantly increased levels in both diabetic mouse myocardium and Angiotensin-II (Ang-II)-induced cardiac fibroblasts (CFs) (Zhou and Yu, 2017). CircRNA_010567 was found to recruit miR-141. The competitive binding of circRNA_010567 and miR-141 released the inhibitory effect of TGF- $\beta 1$, a profibrotic factor, thereby promoting myocardial fibrosis (Zhou and $\mathrm{Yu}, 2017)$. CircRNA_000203 was also revealed to be elevated in diabetic mouse myocardium and Ang-II-induced CFs (Tang et al., 2017). CircRNA_000203 could sponge miR26b-5p to attenuate the inhibition of its targets, Colla2 and CTGF, which are fibrosis-associated proteins. Thus, the 
TABLE 1 | Database for circRNA research.

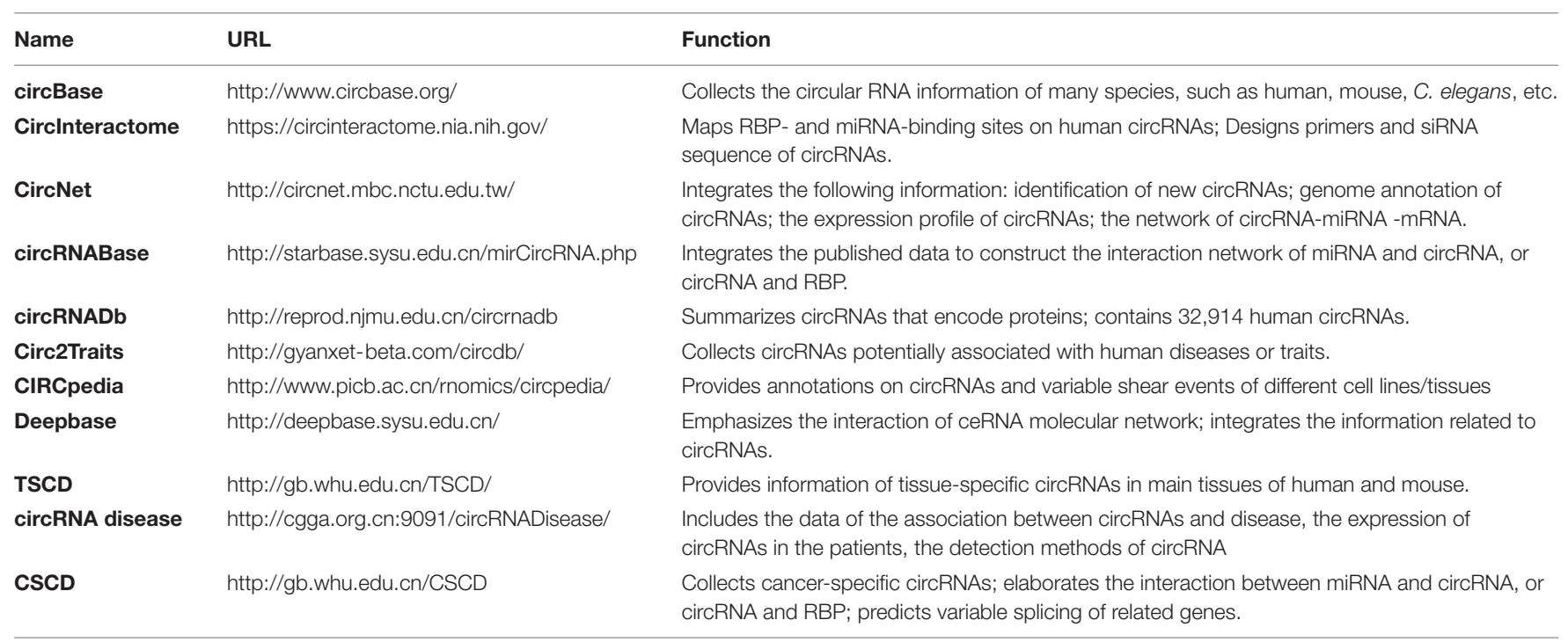

TSCD, tissue specific circRNA database; CSCD, cancer-specific circRNA database; RBP, RNA binding protein.

upregulation of circRNA_000203 may promote the proliferation of CFs (Tang et al., 2017). CircNFIB was downregulated in primary adult CFs treated with TGF- $\beta$ (Zhu et al., 2019). Overexpression of circNFIB attenuates CF proliferation while inhibition of circNFIB promotes CF proliferation, indicating the cardioprotective role of circNFIB (Zhu et al., 2019). CircHIPK3 was upregulated in CFs treated with Ang-II (Ni et al., 2019). CircHIPK3 was revealed to sponge miR-29b-3p which can target fibrosis-associated proteins such as Colla2, Col3a1 and a-SMA. The elevated level of circHIPK3 ultimately enhanced the function of Colla2, Col3a1 and a-SMA, thereby promoting myocardial fibrosis (Ni et al., 2019). In general, circRNA_010567, circRNA_000203 and circHIPK3 are profibrotic while circNFIB is antifibrotic.

\section{Myocardial Injury}

Myocardial injury as well as apoptosis is usually correlated to HF, $\mathrm{MI}$, and ischemia-reperfusion (I/R) injury. CircNCX1 was found to have elevated levels during oxidative stress (Li M. et al., 2018). CircNCX1 could bind to miR-133a-3p and subsequently increase the activity of cell death-inducing protein 1 (CDIP1), inducing apoptosis and I/R injury (Li M. et al., 2018). Circ_0010729 was elucidated to play a role in the injury of human cardiomyocytes induced by oxygen-glucose-deprivation (OGD) (Jin and Chen, 2019). The downregulation of circ_0010729 attenuated the OGDinduced cell injury by activating the mTOR and MEK/ERK pathways (Jin and Chen, 2019). Hsa_circ_0007623 was confirmed to have cardioprotective effects in isoproterenol-induced acute ischemia mice. Hsa_circ_0007623 was able to bind to miR297 and repress the inhibitory effect of miR-297 on VEGF-A, thereby promoting cell proliferation, migration and angiogenesis (Zhang Q. et al., 2020). Wang S. et al. (2019) speculated that circDLGAP4 may regulate cardiomyocyte apoptosis in myocardial I/R injury through targeting miR-143. However, no additional experimental evidence has been reported to date.

\section{Cardiomyopathy}

Cardiomyopathy is a disease with abnormal heart muscles. The muscles are stretched, weakened, or have other structural changes, causing pump difficulties of heart. Most patients with cardiomyopathy will have HF (Molkentin et al., 1998; Aaronson and Sackner-Bernstein, 2006; Rajabi et al., 2007; van Rooij et al., 2008; Authors/Task Force et al., 2014; Wang et al., 2016; Lim et al., 2019). Hypertrophic cardiomyopathy (HCM, cardiac hypertrophy) and dilated cardiomyopathy (DCM, cardiac dilatation) are two common subtypes of cardiomyopathy. When HCM happens, the heart muscles are stretched and become thick, thereby decreasing or blocking the blood flow. In DCM, the heart muscles are weakened, leading to the loss of pumping power of the heart. HRCR could bind to miR-223-5p to decrease its activity and could subsequently upregulate its target, ARC (apoptosis inhibitor with CARD domain), resulting in the inhibition of HCM and HF (Wang et al., 2016). CircSLC8A1 has recently been demonstrated to be the sponge of miR133a (Lim et al., 2019). The knockdown of circSLC8A1 in cardiomyocytes lessened the hypertrophy induced by pressure overload, whereas the overexpression of circSLC8A1 caused HF (Lim et al., 2019). CircRNA_000203 can bind to miR26b-5p and miR-140-3p to increase the expression level of their target gene-GATA4 (Li et al., 2019). The elevated level of GATA4 promotes the occurrence of cardiac hypertrophy (Li et al., 2019). Several circRNAs (ciRNA26, ciRNA261, circRNA1191, circRNA4251, and circRNA6913) were reported to exhibit altered expression in cardiac cells with HCM when they were cultured in high levels and normal levels of Dglucose, respectively (Meng et al., 2019). These circRNAs might be sponges of more than 60 miRNAs, suggesting that they have vital functions in HCM.

RBM20 plays a critical role in the splicing of many cardiac genes, whose mutation will cause aggressive DCM (Brauch et al., 2009). RBM20 can regulate the generation of TTN 
TABLE 2 | CircRNAs with CVD.

\begin{tabular}{|c|c|c|c|c|c|}
\hline CVD type & CircRNAs & Source & Action mechanism & Regulation & References \\
\hline \multirow[t]{6}{*}{ Myocardial infarction } & $\mathrm{CDR} 1_{\text {as }}$ & Mouse myocardial tissue & Sponge miR-7 & Up & Geng et al. (2016) \\
\hline & CircRNA_081881 & Blood & Sponge miR-548 & Down & Deng et al. (2016) \\
\hline & CircRNA MFACR & $\mathrm{A} / \mathrm{R}$ and $\mathrm{I} / \mathrm{R}$ mouse models & Sponge miR-652-3p & Up & Wang et al. (2017) \\
\hline & CircNfix & Mouse heart section & $\begin{array}{l}\text { Dual function as miR-214 sponge } \\
\text { and inducing YBX1 degradation }\end{array}$ & Down & Huang et al. (2019) \\
\hline & CircFndc3b & Mouse hearts & Interact with RBP-FUS & Down & Garikipati et al. (2019) \\
\hline & CircTtc3 & Rat myocardium & Sponge miR-15b-5p & Up & Cai et al. (2019) \\
\hline \multirow[t]{4}{*}{ Myocardial fibrosis } & CircRNA_010567 & Mouse myocardium, cardiac fibroblasts & Sponge miR-141 & Up & Zhou and Yu (2017) \\
\hline & CircRNA_000203 & Mouse myocardium, cardiac fibroblasts & Sponge miR-26b-5p & Up & Tang et al. (2017) \\
\hline & CircNFIB & Mouse heart tissue & Sponge miR-433 & Down & Zhu et al. (2019) \\
\hline & CircHIPK3 & Mouse cardiac fibroblasts & Sponge miR-29b-3p & UP & Ni et al. (2019) \\
\hline \multirow[t]{4}{*}{ Myocardial injury } & CircNCX1 & Mouse cardiomyocytes & Sponge miR-133a-3p & Up & Li M. et al. (2018) \\
\hline & Circ_0010729 & Human cardiomyocytes & Sponge miR-145-5p & Down & Jin and Chen (2019) \\
\hline & Hsa_circ_0007623 & Acute ischemia mice & Sponge miR-297 & Up & Zhang Q. et al. (2020) \\
\hline & CircDLGAP4 & Myocardial ischemia-reperfusion injury & Sponge miR-143 & Up & Wang S. et al. (2019) \\
\hline \multirow[t]{16}{*}{ Cardiomyopathy } & $\mathrm{HRCR}$ & Mouse cardiomyocytes & Sponge miR-223-5p & Down & Wang et al. (2016) \\
\hline & CircSLC8A1 & Mouse cardiomyocytes & Sponge miR-133a & - & Lim et al. (2019) \\
\hline & CircRNA_000203 & NMVCs & $\begin{array}{l}\text { Sponge miR-26b-5p and } \\
\text { miR-140-3p }\end{array}$ & Up & Li et al. (2019) \\
\hline & CiRNA26 & Mouse cardiomyocytes & Sponge several miRNAs & Down & Meng et al. (2019) \\
\hline & CiRNA261 & Mouse cardiomyocytes & Sponge several miRNAs & Up & Meng et al. (2019) \\
\hline & CircRNA1191 & Mouse cardiomyocytes & Sponge several miRNAs & Down & Meng et al. (2019) \\
\hline & CircRNA6913 & Mouse cardiomyocytes & Sponge several miRNAs & Up & Meng et al. (2019) \\
\hline & CircRNA4251 & Mouse cardiomyocytes & Sponge several miRNAs & Down & Meng et al. (2019) \\
\hline & CircRNATTN 1/2/4/5 & DCM patient with a heterozygous mutation in RBM2O (E913K). & Unknown & Down & Khan et al. (2016) \\
\hline & CircSLC8A1 & Human dilated cardiomyopathy & Unknown & Up & Siede et al. (2017) \\
\hline & $\mathrm{CircCHD7}$ & Human dilated cardiomyopathy & Unknown & Up & Siede et al. (2017) \\
\hline & CircATXN10 & Human dilated cardiomyopathy & Unknown & Up & Siede et al. (2017) \\
\hline & CircDNAJC6 & Human dilated cardiomyopathy & Unknown & Down & Siede et al. (2017) \\
\hline & CircSLC8A1 & Heart tissues from DCM patient & Unknown & Up & Lei et al. (2018) \\
\hline & CircAmotl1 & Human neonatal cardiac tissue & Interact with AKT and PDK1 & Up & Zeng Y. et al. (2017) \\
\hline & CircFoxo3 & Mouse heart tissue & $\begin{array}{l}\text { Interact with ID-1, E2F1, FAK, and } \\
\text { HIF1a }\end{array}$ & Up & Du et al. (2017) \\
\hline \multirow[t]{2}{*}{ Aortic aneurysm disease } & Hsa_circ_000595 & Aortic smooth muscle cells & Sponge miR-19a & Up & Zheng et al. (2015) \\
\hline & Hsa_circRNA_101238 & Human aortic segments & Sponge miR-320a & Up & Zou et al. (2017) \\
\hline
\end{tabular}




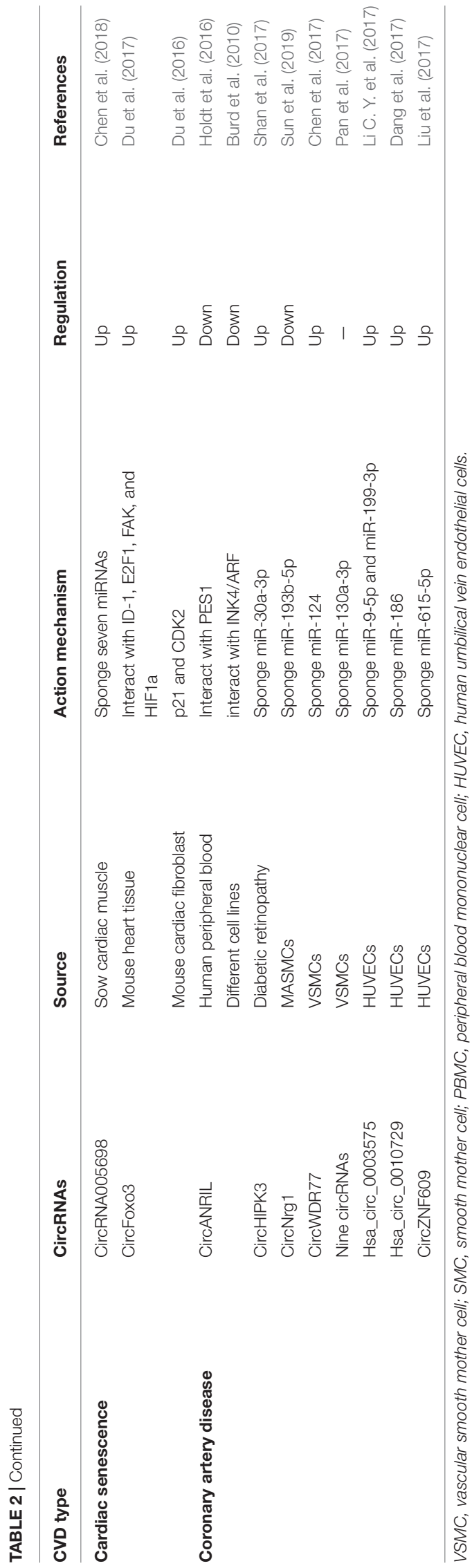

circRNA which might be involved in DCM (Khan et al., 2016). CircSLC8A1, circCHD7, and circATXN10 were found to have elevated expression levels in DCM patients compared with control patients, while circDNAJC6 expression levels were reduced (Siede et al., 2017). The increased expression level of circSLC8A1 was also observed in another study in dilated heart tissue compared with control tissues (Lei et al., 2018). CircAmotll has been reported to bind to PDK1 and AKT1, two cardioprotective molecules (Zeng Y. et al., 2017). This interaction activated AKT1 through phosphorylation and facilitated the nuclear translocation of AKT1 to protect cardiomyocytes in doxorubicin-induced cardiomyopathy (Zeng Y. et al., 2017). CircFoxo3 has been determined to promote doxorubicin-induced ventricular dilatation (Du et al., 2017).

\section{Aortic Aneurysm Disease}

Aortic dissection is the most serious aneurysm disease. Through screening of aortic tissues from patients with aortic dissection aneurysms, Zheng et al. (2015) found an obviously upregulated circRNA, hsa_circ_000595. Hsa_circ_000595 was found to promote apoptosis in vascular smooth mother cells (VSMCs) under hypoxic conditions through upregulating miR-19a expression. Zou et al. (2017) found 162 circRNAs with abnormal expression by microarray analysis of three thoracic aortic dissection (TAD) patients and three control subjects, in which hsa_circRNA_101238 was notably increased. Hsa_circRNA_101238 could sponge miR-320a to inhibit its activity, thereby increasing the levels of its target, MMP9 protein (a TAD related protein) (Zou et al., 2017).

\section{Cardiac Senescence}

Cardiac senescence greatly depresses heart function. Through high throughput RNA-seq, Chen et al. (2018) identified 22 circRNAs with dynamic expression in cardiac muscle during aging. Some of them might regulate the pro-coagulation process. CircRNA005698 was found to be a sponge for seven miRNAs and might be a biomarker for cardiac senescence (Chen et al., 2018). CircFoxo3 was reported to bind to several RBPs (ID-1, E2F1, FAK and HIF1a) and inhibit their activities, thereby promoting cardiomyocyte senescence (Du et al., 2017). CircFoxo3 could also absorb two G1 to S phase transition-related proteins (p21 and CDK2) and suppress their functions in the cell cycle, leading to cell cycle repression (Du et al., 2016).

\section{Hypertension}

Hypertension is a common chronic disease and a major risk factor for CVD. Through profiling of plasma, Wu et al. (2017) identified 59 circRNAs that exhibited altered expression between hypertensive patients and healthy controls. Additionally, a profiling with blood found 351 circRNAs that had different levels from patients with chronic thromboembolic pulmonary hypertension and healthy people (Miao et al., 2017). However, due to the small cohort (five patients and five controls for both studies), all the results in these studies require further validation. Overall, the number of studies on circRNAs in 
TABLE 3 | Circulating circRNAs as diagnostic biomarkers of CVD.

\begin{tabular}{|c|c|c|c|c|}
\hline CVD type & CircRNAs & Source & Regulation & References \\
\hline \multirow[t]{2}{*}{ Myocardial infarction } & MICRA & Peripheral blood & Up & Vausort et al. (2016) \\
\hline & CircRNA_081881 & Plasma & Down & Deng et al. (2016) \\
\hline \multirow[t]{3}{*}{ Congenital heart diseases } & Hsa_circRNA_004183 & Plasma & Down & Wu et al. (2019) \\
\hline & Hsa_circRNA_079265 & Plasma & Down & Wu et al. (2019) \\
\hline & Hsa_circRNA_105039 & Plasma & Down & Wu et al. (2019) \\
\hline Hypertension & Hsa_circ_0037911 & Whole blood & Up & Bao et al. (2018) \\
\hline \multirow[t]{3}{*}{ Cardiomyopathy } & CircDNAJC6 & Serum & Down & Sonnenschein et al. (2019) \\
\hline & CircTMEM56 & Serum & Down & Sonnenschein et al. (2019) \\
\hline & CircMBOAT2 & Serum & Down & Sonnenschein et al. (2019) \\
\hline Heart failure & Hsa_circ_0062960 & Plasma & Up & Sun et al. (2020) \\
\hline \multirow[t]{4}{*}{ Coronary artery disease } & Hsa_circ_0124644 & Peripheral blood & Up & Zhao et al. (2017) \\
\hline & Hsa_circ_0001879 & PBMCs & Up & Wang L. et al. (2019) \\
\hline & Hsa_circ_0004104 & PBMCs & Up & Wang S. et al. (2019) \\
\hline & Hsa_circ_0001445 & Plasma & Down & Vilades et al. (2020) \\
\hline Atrial fibrillation & Hsa_circ_025016 & Plasma & Up & Zhang J. et al. (2018) \\
\hline
\end{tabular}

PBMCs, peripheral blood mononuclear cells.

hypertension remains small. More studies should be conducted to elucidate the mechanisms.

\section{Coronary Artery Disease}

Coronary artery disease is a chronic disease mainly caused by atherosclerosis. miRNAs have been shown to function in all pathogenesis processes of CAD (Zhang $\mathrm{L}$. et al., 2018, 2020), such as endothelial dysfunction, lipid metabolism disorder, proliferation and differentiation of smooth muscle cells (SMCs). Recently, circRNAs have also been found to participate in the development of CAD. CircANRIL was testified to interact with PES1 to suppress pre-rRNA maturation and subsequently restrain the biogenesis of ribosomes, which consequently enhances the stability of anti-atherogenic cells (Holdt et al., 2016). The high level of circANRIL might reduce the severity of CAD (Holdt et al., 2016). Thus, circANRIL plays an atheroprotective role. In addition, circANRIL was also illustrated to play a role in the formation of atherosclerosis by regulating the expression of INK4/ARF (Burd et al., 2010). CircHIPK3 was found to have elevated level in diabetic retinopathy. CircHIPK3 could promote endothelial proliferation and vascular dysfunction through binding to miR-30a-3p which can target VEGFC and WNT2 (Shan et al., 2017). Neuregulin-1 (NRG1) participates in vascular physiopathology (Odiete et al., 2012). CircNrg1 was revealed to sponge miR-193b-5p which could target its host mRNA, Nrg1. The overexpression of circNrg1 led to an elevated level of NRG1, whereas the silencing of circNrg1 decreased the level of NRG1 (Sun et al., 2019). CircWDR77 was determined to be increased in VSMCs treated with high glucose (Chen et al., 2017). CircWDR77 could sponge miR124 to increase the activity of its target, FGF-2 (fibroblast growth factor 2), thereby promoting VSMC proliferation and migration (Chen et al., 2017). Pan et al. (2017) identified 24 circRNAs which were differentially expressed by circRNA microarray. Among these circRNAs, nine circRNAs were found to sponge hsa-miR-130a-3p and then increase the level of TRPM3 which regulates the proliferation and contractility of VSMCs in cooperation with cholesterol (Pan et al., 2017). OxLDL treatment can be employed to induce endothelial cells injury to simulate the pathogenesis of atherosclerosis or CAD. Hsa_circ_0003575 was found to have elevated expression in oxLDL-induced HUVECs (human umbilical vein endothelial cells) (Li C. Y. et al., 2017). The study elaborated that hsa_circ_0003575 could regulate endothelial cells proliferation and angiogenesis probably through interacting with miR-9$5 p$ and miR-199-3p (Li C. Y. et al., 2017). Dang et al. (2017) performed a circRNA microarray in hypoxia-induced HUVECs to identify 36 circRNAs with abnormal expression, and they reported that hsa_circ_0010729 was upregulated. Hsa_circ_0010729 could sponge miR-186 to regulate vascular endothelial cell proliferation and apoptosis via targeting HIF$1 \alpha$. Circular RNA-ZNF609 was reported to have increased expression in HUVECs under high glucose and hypoxia stress, both in vivo and in vitro. CircZNF609 could regulate endothelial cell function by binding to miR-615-5p which targets the transcription factor MEF2A. The knockdown of circZNF609 promoted endothelial cell migration and inhibited endothelial cell apoptosis (Liu et al., 2017).

\section{CircRNAs AS BIOMARKERS FOR CVD}

Currently, a variety of circulating molecules, such as proteins and miRNAs, have been illustrated to have diagnostic potential for CVD. Such proteins as troponins, creatine kinase-MB and myoglobin have been widely used in the clinic. However, these proteins are not specific and are not applicable for the early diagnosis. Additionally, these proteins are easily influenced by such factors as the heart-associated diseases, medication, patient genetic background, and age (Chen et al., 2008; Lawrie et al., 2008). Therefore, protein biomarkers have limited diagnostic value. Circulating miRNAs have been elaborated to have high 
specificity and strong potential for early diagnosis. However, circulating miRNAs have not been applied in the clinic due to their low content and time-consuming detection (Zhang L. et al., 2018). Circulating circRNAs have many features resembling circulating miRNAs including high stability, sensitivity and specificity, which are essential for biomarkers. Meanwhile, the circulating levels of circRNAs are not low, and some circRNAs even have high content, making detection easier. Many studies have revealed the considerable potential of circulating circRNAs as novel and promising biomarkers for the early diagnosis of CVD (Table 3 and Figure 2).

CircZNF609 (MICRA) had lower levels in the peripheral blood of MI patients than in healthy controls (Vausort et al., 2016). Circulating MICRA was demonstrated to have a high value of predicting left ventricular dysfunction (Vausort et al., 2016). CircRNA_081881 was downregulated in the plasma of AMI patients and might be a promising target for AMI diagnosis and therapy (Deng et al., 2016). The level of hsa_circ_0124644 was increased in the peripheral blood of CAD patients and was found to have a significant association with CAD. Receiver operating characteristic (ROC) analysis revealed that circulating hsa_circ_0124644 might be a potential diagnostic biomarker for CAD (Zhao et al., 2017). Hsa_circ_0001879 and hsa_circ_0004104 were found to have increased levels in the peripheral blood mononuclear cells (PBMCs) of CAD patients (Wang L. et al., 2019). ROC analysis revealed their high accuracy in the diagnosis of CAD. Furthermore, the combination of hsa_circ_0001879, hsa_circ_0004104 and CAD risk factors had the highest value to discriminate CAD patients from healthy controls (Wang L. et al., 2019). Atrial fibrillation (AF) is a common complication after coronary artery bypass grafting (CABG) (Maesen et al., 2012). Hsa_circ_025016 was upregulated in the plasma of patients with new-onset $\mathrm{AF}$ after isolated off-pump CABG. ROC analysis revealed a high diagnostic value (Zhang J. et al., 2018). The analysis with a large validation cohort confirmed the diagnostic power of hsa_circ_025016 (Zhang J. et al., 2018). All results indicated that hsa_circRNA_025016 might be a promising biomarker for the prediction of new-onset AF after isolated off-pump CABG (Zhang J. et al., 2018). Sun et al. (2020) performed circRNA microarrays and found significantly upregulated plasma levels of hsa_circ_0112085, hsa_circ_0062960, hsa_circ_0053919 and hsa_circ_0014010 in HF patients. ROC analysis revealed that hsa_circ_0062960 had great potential to be a diagnostic biomarker of HF (Sun et al., 2020). A study using whole blood revealed that the hsa_circ_0037911 level was significantly increased in hypertensive patients in contrast to the control group (Bao et al., 2018). Another study revealed reduced expression levels of circRNAs (DNAJC6, TMEM56 and MBOAT2) in the serum of patients with HCM (Sonnenschein et al., 2019). All three circRNAs had high discrimination power between HCM patients and the control cohort. Moreover, circTMEM56 and circDNAJC6 could be indicators of disease severity in patients with HCM (Sonnenschein et al., 2019). Wu et al. (2019) reported three notably downregulated circRNAs (hsa_circRNA_004183, hsa_circRNA_079265 and hsa_circRNA_105039) in the plasma of children with congenital heart diseases (CHD) and employed
ROC analyses to determine their potential to be biomarkers. They found the great potential of three circRNAs as novel non-invasive diagnostic biomarkers for CHD (Wu et al., 2019). Hsa_circ_0001445 was shown to have lower plasma levels in CAD patients than in the control group (Vilades et al., 2020). Hsa_circ_0001445 is secreted into circulation through being packaged in extracellular vesicles of coronary SMCs (Vilades et al., 2020). The coronary atherosclerotic condition abolished the association of hsa_circ_0001445 and vesicles, leading to the downregulation of plasma hsa_circ_0001445 (Vilades et al., 2020). Therefore, hsa_circ_0001445 might be considered an effective and novel predictor of CAD (Vilades et al., 2020). In general, these studies have illuminated the potential role of circulating circRNAs as biomarkers for the diagnosis and prognosis of CVD.

\section{CONCLUSION AND FUTURE PERSPECTIVES}

Base on our exploration, the results from a variety of studies have confirmed that circRNAs can participate in the pathogenesis of CVD mainly through acting as miRNA sponges and interacting with RBPs. CircRNAs are widely distributed in different tissues and have tissue- and developmental stage-specific expression. In addition, circRNAs are stable and abundantly present in the circulatory system. Therefore, circRNAs might be promising biomarkers for the diagnosis of CVD, and accumulating research has confirmed this possibility. The clinical use of circRNAs as diagnostic biomarkers will greatly facilitate the prevention and treatment of CVD. However, there are some problems that should be solved before clinical application of circRNAs.

First, there is no generally accepted methodology on the measurement procedures of circulating circRNAs, which might result in the lack of consistency in various studies. Hence, a standardized methodology should be formulated before clinical use. Second, the sample sizes are small in most studies. The insufficient samples might lead to deviation in the test results. A larger cohort is necessary for correct conclusions. Finally, despite these findings, the underlying mechanisms of the functions of many circulating circRNAs have not been elucidated, and our knowledge is still insufficient, which represents a considerable obstacle to clinical application. More and deeper studies should be performed to explore the potential molecular mechanisms.

In summary, studies have confirmed that circRNAs are closely involved in the progression of CVD and might be promising biomarkers for CVD. These findings may provide a new avenue for the prevention, diagnosis and therapeutic intervention of CVD in the future.

\section{AUTHOR CONTRIBUTIONS}

LZ and YZhang drafted the manuscript. YZhao and HD edited the manuscript. YW revised the manuscript. PL and LZ conceived the idea and framework of the review and made 
the final proofreading. All authors read and approved the final manuscript.

\section{FUNDING}

This work was supported by the National Natural Science Foundation of China (Grant Numbers: 91849209 and 31430041)

\section{REFERENCES}

Aaronson, K. D., and Sackner-Bernstein, J. (2006). Risk of death associated with nesiritide in patients with acutely decompensated heart failure. JAMA 296, 1465-1466. doi: 10.1001/jama.296.12.1465

Abdelmohsen, K., Panda, A. C., Munk, R., Grammatikakis, I., Dudekula, D. B., De, S., et al. (2017). Identification of HuR target circular RNAs uncovers suppression of PABPN1 translation by CircPABPN1. RNA Biol. 14, 361-369. doi: 10.1080/15476286.2017.1279788

AbouHaidar, M. G., Venkataraman, S., Golshani, A., Liu, B. L., and Ahmad, T. (2014). Novel coding, translation, and gene expression of a replicating covalently closed circular RNA of 220 nt. Proc. Natl. Acad. Sci. U.S.A. 111, 14542-14547. doi: 10.1073/pnas.1402814111

Asha, P. K., Blouin, R. T., Zaniewski, R., and Deutscher, M. P. (1983). Ribonuclease BN: identification and partial characterization of a new tRNA processing enzyme. Proc. Natl. Acad. Sci. U.S.A. 80, 3301-3304. doi: 10.1073/pnas.80.11. 3301

Ashwal-Fluss, R., Meyer, M., Pamudurti, N. R., Ivanov, A., Bartok, O., Hanan, M., et al. (2014). circRNA biogenesis competes with pre-mRNA splicing. Mol. Cell 56, 55-66. doi: 10.1016/j.molcel.2014.08.019

Aufiero, S., Reckman, Y. J., Pinto, Y. M., and Creemers, E. E. (2019). Circular RNAs open a new chapter in cardiovascular biology. Nat. Rev. Cardiol. 16, 503-514.

Authors/Task Force, M., Elliott, P. M., Anastasakis, A., Borger, M. A., Borggrefe, M., Cecchi, F., et al. (2014). 2014 ESC Guidelines on diagnosis and management of hypertrophic cardiomyopathy: the Task Force for the Diagnosis and Management of Hypertrophic Cardiomyopathy of the European Society of Cardiology (ESC). Eur. Heart J. 35, 2733-2779. doi: 10.1093/eurheartj/ehu284

Bao, X., Zheng, S., Mao, S., Gu, T., Liu, S., Sun, J., et al. (2018). A potential risk factor of essential hypertension in case-control study: Circular RNA hsa_circ_0037911. Biochem. Biophys. Res. Commun. 498, 789-794. doi: 10.1016/ j.bbrc.2018.03.059

Brauch, K. M., Karst, M. L., Herron, K. J., de Andrade, M., Pellikka, P. A., Rodeheffer, R. J., et al. (2009). Mutations in ribonucleic acid binding protein gene cause familial dilated cardiomyopathy. J. Am. Coll. Cardiol. 54, 930-941. doi: 10.1016/j.jacc.2009.05.038

Burd, C. E., Jeck, W. R., Liu, Y., Sanoff, H. K., Wang, Z., and Sharpless, N. E. (2010). Expression of linear and novel circular forms of an INK4/ARF-associated noncoding RNA correlates with Atherosclerosis risk. PLoS Genet. 6:e1001233. doi: 10.1371/journal.pgen.1001233

Cai, L., Qi, B., Wu, X., Peng, S., Zhou, G., Wei, Y., et al. (2019). Circular RNA Ttc3 regulates cardiac function after myocardial infarction by sponging miR-15b. J. Mol. Cell Cardiol. 130, 10-22. doi: 10.1016/j.yjmcc.2019.03.007

Capel, B., Swain, A., Nicolis, S., Hacker, A., Walter, M., Koopman, P., et al. (1993). Circular transcripts of the testis-determining gene Sry in adult mouse testis. Cell 73, 1019-1030. doi: 10.1016/0092-8674(93)90279-y

Chen, C. Y., and Sarnow, P. (1995). Initiation of protein synthesis by the eukaryotic translational apparatus on circular RNAs. Science 268, 415-417. doi: 10.1126/ science.7536344

Chen, J., Zou, Q., Lv, D., Wei, Y., Raza, M. A., Chen, Y., et al. (2018). Comprehensive transcriptional landscape of porcine cardiac and skeletal muscles reveals differences of aging. Oncotarget 9, 1524-1541. doi: 10.18632/ oncotarget. 23290

Chen, J. J., Cui, L. Q., Yuan, J. L., Zhang, Y. Q., and Sang, H. J. (2017). Circular RNA WDR77 target FGF-2 to regulate vascular smooth muscle cells proliferation and migration by sponging miR-124. Biochem. Biophys. Res. Commun. 494, 126-132. doi: 10.1016/j.bbrc.2017.10.068 and Innovative Talent Program of Qingdao City, China (18-12-6-zhc).

\section{ACKNOWLEDGMENTS}

The authors would like to thank Liang $\mathrm{Xu}$ for the help in the manuscript revision.

Chen, X., Ba, Y., Ma, L., Cai, X., Yin, Y., Wang, K., et al. (2008). Characterization of microRNAs in serum: a novel class of biomarkers for diagnosis of cancer and other diseases. Cell Res. 18, 997-1006. doi: 10.1038/cr.2008.282

Cocquerelle, C., Daubersies, P., Majerus, M. A., Kerckaert, J. P., and Bailleul, B. (1992). Splicing with inverted order of exons occurs proximal to large introns. EMBO J. 11, 1095-1098.

Cocquerelle, C., Mascrez, B., Hetuin, D., and Bailleul, B. (1993). Mis-splicing yields circular RNA molecules. FASEB J. 7, 155-160. doi: 10.1096/fasebj.7.1.7678559

Conn, S. J., Pillman, K. A., Toubia, J., Conn, V. M., Salmanidis, M., Phillips, C. A., et al. (2015). The RNA binding protein quaking regulates formation of circRNAs. Cell 160, 1125-1134. doi: 10.1016/j.cell.2015.02.014

Dang, R. Y., Liu, F. L., and Li, Y. (2017). Circular RNA hsa_circ_0010729 regulates vascular endothelial cell proliferation and apoptosis by targeting the miR186/HIF-1alpha axis. Biochem. Biophys. Res. Commun. 490, 104-110. doi: 10. 1016/j.bbrc.2017.05.164

Deng, Y. Y., Zhang, W. P., She, J. Q., Zhang, L. S., Chen, T., Zhou, J., et al. (2016). Circular RNA related to PPAR gamma function as ceRNA of microRNA in human acute myocardial infarction. J Am. Coll Cardiol. 68, C51-C52. doi: 10.1016/j.jacc.2016.07.189

Du, W. W., Yang, W., Chen, Y., Wu, Z. K., Foster, F. S., Yang, Z., et al. (2017). Foxo3 circular RNA promotes cardiac senescence by modulating multiple factors associated with stress and senescence responses. Eur. Heart J. 38, 1402-1412. doi: 10.1093/eurheartj/ehw001

Du, W. W., Yang, W., Liu, E., Yang, Z., Dhaliwal, P., and Yang, B. B. (2016). Foxo3 circular RNA retards cell cycle progression via forming ternary complexes with p21 and CDK2. Nucl. Acids Res. 44, 2846-2858. doi: 10.1093/nar/gkw027

Fan, X., Weng, X., Zhao, Y., Chen, W., Gan, T., and Xu, D. (2017). Circular RNAs in Cardiovascular Disease: An Overview. Biomed. Res. Int. 2017:5135781. doi: 10.1155/2017/5135781

Garikipati, V. N. S., Verma, S. K., Cheng, Z. J., Liang, D. M., Truongcao, M. M., Cimini, M., et al. (2019). Circular RNA CircFndc3b modulates cardiac repair after myocardial infarction via FUS/VEGF-A axis. Nat. Commun. 10:4317.

Geng, H. H., Li, R., Su, Y. M., Xiao, J., Pan, M., Cai, X. X., et al. (2016). The circular RNA Cdrlas promotes myocardial infarction by mediating the regulation of miR-7a on its target genes expression. PLoS One 11:e0151753. doi: 10.1371/ journal.pone.0151753

Gupta, S. K., Garg, A., Bar, C., Chatterjee, S., Foinquinos, A., Milting, H., et al. (2018). Quaking inhibits doxorubicin-mediated cardiotoxicity through regulation of cardiac circular RNA expression. Circ. Res. 122, 246-254. doi: 10.1161/Circresaha.117.311335

Hansen, T. B., Jensen, T. I., Clausen, B. H., Bramsen, J. B., Finsen, B., Damgaard, C. K., et al. (2013a). Natural RNA circles function as efficient microRNA sponges. Nature 495, 384-388. doi: 10.1038/nature11993

Hansen, T. B., Kjems, J., and Damgaard, C. K. (2013b). Circular RNA and miR-7 in cancer. Cancer Res. 73, 5609-5612.

Hansen, T. B., Veno, M. T., Damgaard, C. K., and Kjems, J. (2016). Comparison of circular RNA prediction tools. Nucl. Acids Res. 44:e58. doi: 10.1093/nar/ gkv1458

Holdt, L. M., Stahringer, A., Sass, K., Pichler, G., Kulak, N. A., Wilfert, W., et al. (2016). Circular non-coding RNA ANRIL modulates ribosomal RNA maturation and atherosclerosis in humans. Nat. Commun. 7:12429. doi: 10. 1038/ncomms 12429

Huang, S. L., Li, X. Z., Zheng, H., Si, X. Y., Li, B., Wei, G. Q., et al. (2019). Loss of super-enhancer-regulated circRNA Nfix induces cardiac regeneration after myocardial infarction in adult mice. Circulation 139, 2857-2876. doi: 10.1161/ Circulationaha.118.038361 
Jakobi, T., Czaja-Hasse, L. F., Reinhardt, R., and Dieterich, C. (2016). Profiling and validation of the circular RNA repertoire in adult murine hearts. Genom. Proteom. Bioinf. 14, 216-223. doi: 10.1016/j.gpb.2016.02.003

Jeck, W. R., and Sharpless, N. E. (2014). Detecting and characterizing circular RNAs. Nat. Biotechnol. 32, 453-461. doi: 10.1038/nbt.2890

Jeck, W. R., Sorrentino, J. A., Wang, K., Slevin, M. K., Burd, C. E., Liu, J. Z., et al. (2013). Circular RNAs are abundant, conserved, and associated with ALU repeats. RNA 19, 141-157. doi: 10.1261/rna.035667.112

Jin, Q. F., and Chen, Y. Y. (2019). Silencing circular RNA circ_0010729 protects human cardiomyocytes from oxygen-glucose deprivation-induced injury by up-regulating microRNA-145-5p. Mol. Cell Biochem. 462, 185-194.

Khan, M. A., Reckman, Y. J., Aufiero, S., van den Hoogenhof, M. M., van der Made, I., Beqqali, A., et al. (2016). RBM20 regulates circular RNA production from the titin gene. Circ. Res. 119, 996-1003. doi: 10.1161/CIRCRESAHA.116.309568

Kolakofsky, D. (1976). Isolation and characterization of Sendai virus DI-RNAs. Cell 8, 547-555.

Kos, A., Dijkema, R., Arnberg, A. C., van der Meide, P. H., and Schellekens, H. (1986). The hepatitis delta (delta) virus possesses a circular RNA. Nature 323, 558-560. doi: 10.1038/323558a0

Kramer, M. C., Liang, D., Tatomer, D. C., Gold, B., March, Z. M., Cherry, S., et al. (2015). Combinatorial control of Drosophila circular RNA expression by intronic repeats, hnRNPs, and SR proteins. Genes Dev. 29, 2168-2182. doi: $10.1101 /$ gad.270421.115

Lawrie, C. H., Gal, S., Dunlop, H. M., Pushkaran, B., Liggins, A. P., Pulford, K., et al. (2008). Detection of elevated levels of tumour-associated microRNAs in serum of patients with diffuse large B-cell lymphoma. Br. J. Haematol. 141, 672-675. doi: 10.1111/j.1365-2141.2008.07077.x

Legnini, I., Di Timoteo, G., Rossi, F., Morlando, M., Briganti, F., Sthandier, O., et al. (2017). Circ-ZNF609 is a circular RNA that can be translated and functions in myogenesis. Mol. Cell 66, 22-37. doi: 10.1016/j.molcel.2017.02.017

Lei, W., Feng, T., Fang, X., Yu, Y., Yang, J., Zhao, Z. A., et al. (2018). Signature of circular RNAs in human induced pluripotent stem cells and derived cardiomyocytes. Stem. Cell Res. Ther. 9:56.

Li, C. Y., Ma, L., and Yu, B. (2017). Circular RNA hsa_circ_0003575 regulates oxLDL induced vascular endothelial cells proliferation and angiogenesis. Biomed. Pharmacother. 95, 1514-1519. doi: 10.1016/j.biopha.2017.09.064

Li, H., Xu, J. D., Fang, X. H., Zhu, J. N., Yang, J., Pan, R., et al. (2019). Circular RNA circRNA_000203 aggravates cardiac hypertrophy via suppressing miR26b-5p and miR-140-3p binding to Gata4. Cardiovasc. Res. 116, 1323-1334. doi: 10. $1093 / \mathrm{cvr} / \mathrm{cvz} 215$

Li, M., Ding, W., Tariq, M. A., Chang, W., Zhang, X., Xu, W., et al. (2018). A circular transcript of ncxl gene mediates ischemic myocardial injury by targeting miR-133a-3p. Theranostics 8, 5855-5869. doi: 10.7150/thno.27285

Li, Y., Chen, B., and Huang, S. (2018). Identification of circRNAs for miRNA Targets by Argonaute2 RNA immunoprecipitation and luciferase screening assays. Methods Mol. Biol. 1724, 209-218. doi: 10.1007/978-1-4939-7562-4_17

Li, Y. S., Zhang, J. W., Huo, C. Q., Ding, N., Li, J. Y., Xiao, J., et al. (2017). Dynamic organization of lncrna and circular rna regulators collectively controlled cardiac differentiation in humans. Ebiomedicine 24, 137-146. doi: 10.1016/j.ebiom. 2017.09.015

Li, Z. Y., Huang, C., Bao, C., Chen, L., Lin, M., Wang, X. L., et al. (2015). Exonintron circular RNAs regulate transcription in the nucleus. Nat. Struct. Mol. Biol. 22, 256-264. doi: 10.1038/nsmb.2959

Liang, W. C., Wong, C. W., Liang, P. P., Shi, M., Cao, Y., Rao, S. T., et al. (2019). Translation of the circular RNA circ-catenin promotes liver cancer cell growth through activation of the Wnt pathway. Genome Biol 20:84.

Lim, T. B., Aliwarga, E., Luu, T. D. A., Li, Y. P., Ng, S. L., Annadoray, L., et al. (2019). Targeting the highly abundant circular RNA circSlc8al in cardiomyocytes attenuates pressure overload induced hypertrophy. Cardiovasc Res 115, 19982007. doi: $10.1093 / \mathrm{cvr} / \mathrm{cvz} 130$

Lim, T. B., Lavenniah, A., and Foo, R. S. (2020). Circles in the heart and cardiovascular system. Cardiovasc. Res. 116, 269-278. doi: 10.1093/cvr/cvz227

Liu, C., Yao, M. D., Li, C. P., Shan, K., Yang, H., Wang, J. J., et al. (2017). Silencing of circular RNA-ZNF609 ameliorates vascular endothelial dysfunction. Theranostics 7, 2863-2877. doi: 10.7150/thno.19353

Liu, L., Liu, F. B., Huang, M., Xie, K., Xie, Q. S., Liu, C. H., et al. (2019). Circular RNA ciRS-7 promotes the proliferation and metastasis of pancreatic cancer by regulating miR-7-mediated EGFR/STAT3 signaling pathway. Hepatob. Pancreat. Dis. Int. 18, 580-586. doi: 10.1016/j.hbpd.2019.03.003

Maesen, B., Nijs, J., Maessen, J., Allessie, M., and Schotten, U. (2012). Postoperative atrial fibrillation: a maze of mechanisms. Europace 14, 159-174. doi: 10.1093/europace/eur208

Memczak, S., Jens, M., Elefsinioti, A., Torti, F., Krueger, J., Rybak, A., et al. (2013). Circular RNAs are a large class of animal RNAs with regulatory potency. Nature 495, 333-338. doi: 10.1038/nature11928

Meng, Z. Y., Chen, C., Cao, H. L., Wang, J. Y., and Shen, E. (2019). Whole transcriptome sequencing reveals biologically significant RNA markers and related regulating biological pathways in cardiomyocyte hypertrophy induced by high glucose. J. Cell Biochem. 120, 1018-1027. doi: 10.1002/jcb.27546

Meyer, K. D., Patil, D. P., Zhou, J., Zinoviev, A., Skabkin, M. A., Elemento, O., et al. (2015). $5^{\prime}$. UTR m(6)A promotes cap-independent translation. Cell 163, 999-1010. doi: 10.1016/j.cell.2015.10.012

Miao, R., Wang, Y., Wan, J., Leng, D., Gong, J., Li, J., et al. (2017). Microarray expression profile of circular RNAs in chronic thromboembolic pulmonary hypertension. Medicine 96:e7354. doi: 10.1097/MD.0000000000007354

Molkentin, J. D., Lu, J. R., Antos, C. L., Markham, B., Richardson, J., Robbins, J., et al. (1998). A calcineurin-dependent transcriptional pathway for cardiac hypertrophy. Cell 93, 215-228.

Ni, H., Li, W., Zhuge, Y., Xu, S., Wang, Y., Chen, Y., et al. (2019). Inhibition of circHIPK3 prevents angiotensin II-induced cardiac fibrosis by sponging miR-29b-3p. Int. J. Cardiol. 292, 188-196. doi: 10.1016/j.ijcard.2019.04.006

Nigro, J. M., Cho, K. R., Fearon, E. R., Kern, S. E., Ruppert, J. M., Oliner, J. D., et al. (1991). Scrambled exons. Cell 64, 607-613. doi: 10.1016/0092-8674(91)90244-s

Odiete, O., Hill, M. F., and Sawyer, D. B. (2012). Neuregulin in cardiovascular development and disease. Circ. Res. 111, 1376-1385. doi: 10.1161/CIRCRESAHA.112.267286

Pamudurti, N. R., Bartok, O., Jens, M., Ashwal-Fluss, R., Stottmeister, C., Ruhe, L., et al. (2017). Translation of CircRNAs. Mol. Cell 66, 9-21e27. doi: 10.1016/j. molcel.2017.02.021

Pan, H., Li, T., Jiang, Y., Pan, C., Ding, Y., Huang, Z., et al. (2018). Overexpression of circular RNA ciRS-7 Abrogates the tumor suppressive effect of miR-7 on gastric cancer via PTEN/PI3K/AKT signaling pathway. J. Cell Biochem. 119, 440-446. doi: 10.1002/jcb.26201

Pan, R. Y., Liu, P., Zhou, H. T., Sun, W. X., Song, J., Shu, J., et al. (2017). Circular RNAs promote TRPM3 expression by inhibiting hsa-miR-130a-3p in coronary artery disease patients. Oncotarget 8, 60280-60290. doi: 10.18632/oncotarget. 19941

Perriman, R., and Ares, M. (1998). ). Circular mRNA can direct translation of extremely long repeating-sequence proteins in vivo. RNA 4, 1047-1054. doi: 10.1017/S135583829898061x

Rajabi, M., Kassiotis, C., Razeghi, P., and Taegtmeyer, H. (2007). Return to the fetal gene program protects the stressed heart: a strong hypothesis. Heart Fail Rev. $12,331-343$.

Rybak-Wolf, A., Stottmeister, C., Glazar, P., Jens, M., Pino, N., Giusti, S., et al. (2015). Circular RNAs in the mammalian brain are highly abundant, conserved, and dynamically expressed. Mol. Cell 58, 870-885. doi: 10.1016/j.molcel.2015. 03.027

Schroeder, R., Breitenbach, M., and Schweyen, R. J. (1983). Mitochondrial circular RNAs are absent in sporulating cells of Saccharomyces cerevisiae. Nucl. Acids Res 11, 1735-1746. doi: 10.1093/nar/11.6.1735

Shan, K., Liu, C., Liu, B. H., Chen, X., Dong, R., Liu, X., et al. (2017). Circular noncoding RNA HIPK3 mediates retinal vascular dysfunction in diabetes mellitus. Circulation 136, 1629-1642. doi: 10.1161/CIRCULATIONAHA.117. 029004

Siede, D., Rapti, K., Gorska, A. A., Katus, H. A., Altmuller, J., Boeckel, J. N., et al. (2017). Identification of circular RNAs with host gene-independent expression in human model systems for cardiac differentiation and disease. J. Mol. Cell Cardiol. 109, 48-56. doi: 10.1016/j.yjmcc.2017.06.015

Sonnenschein, K., Wilczek, A. L., de Gonzalo-Calvo, D., Pfanne, A., Derda, A. A., Zwadlo, C., et al. (2019). Serum circular RNAs act as blood-based biomarkers for hypertrophic obstructive cardiomyopathy. Sci. Rep. 9:20350.

Sun, Y., Jiang, X., Lv, Y., Liang, X., Zhao, B., Bian, W., et al. (2020). Circular rna expression profiles in plasma from patients with heart failure related to platelet activity. Biomolecules 10:187. doi: 10.3390/biom10020187 
Sun, Y., Zhang, S. L., Yue, M. M., Li, Y., Bi, J., and Liu, H. R. (2019). Angiotensin II inhibits apoptosis of mouse aortic smooth muscle cells through regulating the circNRG-1/miR-193b-5p/NRG-1 axis. Cell. Death Dis. 10:362.

Suzuki, H., Zuo, Y. H., Wang, J. H., Zhang, M. Q., Malhotra, A., and Mayeda, A. (2006). Characterization of RNase R-digested cellular RNA source that consists of lariat and circular RNAs from pre-mRNA splicing. Nucl. Acids Res. 34:e63. doi: $10.1093 / \mathrm{nar} / \mathrm{gkl} 151$

Szabo, L., and Salzman, J. (2016). Detecting circular RNAs: bioinformatic and experimental challenges. Nat. Rev. Genet. 17, 679-692. doi: 10.1038/nrg. 2016.114

Tan, W. L., Lim, B. T., Anene-Nzelu, C. G., Ackers-Johnson, M., Dashi, A., See, K., et al. (2017). A landscape of circular RNA expression in the human heart. Cardiov. Res. 113, 298-309. doi: 10.1093/cvr/cvw250

Tang, C. M., Zhang, M., Huang, L., Hu, Z. Q., Zhu, J. N., Xiao, Z., et al. (2017). CircRNA_000203 enhances the expression of fibrosis-associated genes by derepressing targets of miR-26b-5p, Colla2 and CTGF, in cardiac fibroblasts. Sci. Rep. 7:40342. doi: 10.1038/srep40342

Tay, Y., Rinn, J., and Pandolfi, P. P. (2014). The multilayered complexity of ceRNA crosstalk and competition. Nature 505, 344-352. doi: 10.1038/nature12986

van Rooij, E., Marshall, W. S., and Olson, E. N. (2008). Toward microRNA-based therapeutics for heart disease: the sense in antisense. Circ. Res. 103, 919-928. doi: 10.1161/CIRCRESAHA.108.183426

Vausort, M., Salgado-Somoza, A., Zhang, L., Leszek, P., Scholz, M., Teren, A., et al. (2016). Myocardial infarction-associated circular RNA predicting left ventricular dysfunction. J. Am. Coll. Cardiol. 68, 1247-1248. doi: 10.1016/j.jacc. 2016.06.040

Vilades, D., Martinez-Camblor, P., Ferrero-Gregori, A., Bar, C., Lu, D. C., Xiao, K., et al. (2020). Plasma circular RNA hsa_circ_0001445 and coronary artery disease: performance as a biomarker. Faseb J. 34, 4403-4414. doi: 10.1096/fj. 201902507R

Wang, K., Gan, T. Y., Li, N., Liu, C. Y., Zhou, L. Y., Gao, J. N., et al. (2017). Circular RNA mediates cardiomyocyte death via miRNA-dependent upregulation of MTP18 expression. Cell Death Differ. 24, 1111-1120. doi: 10.1038/cdd.2017.61

Wang, K., Long, B., Liu, F., Wang, J. X., Liu, C. Y., Zhao, B., et al. (2016). A circular RNA protects the heart from pathological hypertrophy and heart failure by targeting miR-223. Eur. Heart J 37, 2602-2611. doi: 10.1093/eurheartj/ehv713

Wang, L., Shen, C., Wang, Y., Zou, T., Zhu, H., Lu, X., et al. (2019). Identification of circular RNA Hsa_circ_0001879 and Hsa_circ_0004104 as novel biomarkers for coronary artery disease. Atherosclerosis 286, 88-96. doi: 10.1016/j.atherosclerosis.2019.05.006

Wang, S., Chen, J. Y., Yu, W. Q., and Deng, F. (2019). Circular RNA DLGAP4 ameliorates cardiomyocyte apoptosis through regulating BCL2 via targeting miR-143 in myocardial ischemia-reperfusion injury. Int. J. Cardiol. 279, 147147. doi: 10.1016/j.ijcard.2018.09.023

Werfel, S., Nothjunge, S., Schwarzmayr, T., Strom, T. M., Meitinger, T., and Engelhardt, S. (2016). Characterization of circular RNAs in human, mouse and rat hearts. J. Mol. Cell Cardiol. 98, 103-107. doi: 10.1016/j.yjmcc.2016.07.007

Wesselhoeft, R. A., Kowalski, P. S., and Anderson, D. G. (2018). Engineering circular RNA for potent and stable translation in eukaryotic cells. Nat. Commun. 9:2629.

Wu, J. H., Li, J. Q., Liu, H., Yin, J. W., Zhang, M. J., Yu, Z. B., et al. (2019). Circulating plasma circular RNAs as novel diagnostic biomarkers for congenital heart disease in children. J. Clin. Lab. Anal. 33:e22998. doi: 10.1002/jcla.22998

Wu, N., Jin, L., and Cai, J. (2017). Profiling and bioinformatics analyses reveal differential circular RNA expression in hypertensive patients. Clin. Exp. Hypertens 39, 454-459. doi: 10.1080/10641963.2016.1273944

Xu, T. Y., Wu, J., Han, P., Zhao, Z. M., and Song, X. F. (2017). Circular RNA expression profiles and features in human tissues: a study using RNA-seq data. Bmc Genom. 18:680. doi: 10.1186/s12864-017-4029-23

Yang, Y., Fan, X., Mao, M., Song, X., Wu, P., Zhang, Y., et al. (2017). Extensive translation of circular RNAs driven by N(6)-methyladenosine. Cell Res. 27, 626-641. doi: 10.1038/cr.2017.31

Yang, Y., Gao, X., Zhang, M., Yan, S., Sun, C., Xiao, F., et al. (2018). Novel role of FBXW7 circular RNA in repressing glioma tumorigenesis. J. Natl. Cancer Inst. 110:435. doi: 10.1093/jnci/djx166

Yang, Z. G., Awan, F. M., Du, W. W., Zeng, Y., Lyu, J., Wu, et al. (2017). The circular RNA interacts with STAT3, increasing its nuclear translocation and wound repair by modulating Dnmt3a and miR-17 function. Mol. Ther. 25, 2062-2074. doi: 10.1016/j.ymthe.2017.05.022

Yang, Z. G., Guo, X. B., Li, G. M., Shi, Y. L., and Li, L. P. (2016). Long noncoding RNAs as potential biomarkers in gastric cancer: opportunities and challenges. Cancer Lett. 371, 62-70. doi: 10.1016/j.canlet.2015.11.011

Zeng, X. X., Lin, W., Guo, M. Z., and Zou, Q. (2017). A comprehensive overview and evaluation of circular RNA detection tools. PLos Comp. Biol. 13:e1005420. doi: 10.1371/journal.pcbi.1005420

Zeng, Y., Du, W. W., Wu, Y., Yang, Z., Awan, F. M., Li, X., et al. (2017). A circular RNA binds to and activates AKT phosphorylation and nuclear localization reducing apoptosis and enhancing cardiac repair. Theranostics 7, 3842-3855. doi: 10.7150/thno.19764

Zhang, J., Xu, Y. L., Xu, S., Liu, Y., Yu, L. M., Li, Z., et al. (2018). Plasma circular RNAs, Hsa_circRNA_025016, predict postoperative atrial fibrillation after isolated off-pump coronary artery bypass grafting. J. Am. Heart Assoc. 7:e006642. doi: 10.1161/JAHA.117.006642

Zhang, L., Zhang, Y., Xue, S., Ding, H., Wang, Y., Qi, H. Z., et al. (2020). Clinical significance of circulating microRNAs as diagnostic biomarkers for coronary artery disease. J. Cell Mol. Med. 24, 1146-1150. doi: 10.1111/jcmm.14802

Zhang, L., Zhang, Y., Zhao, Y. F., Wang, Y., Ding, H., Xue, S., et al. (2018). Circulating miRNAs as biomarkers for early diagnosis of coronary artery disease. Expert Opin Ther Pat 28, 591-601. doi: 10.1080/13543776.2018. 1503650

Zhang, Q., Sun, W. X., Han, J., Cheng, S. Y., Yu, P., Shen, L., et al. (2020). The circular RNA hsa_circ_0007623 acts as a sponge of microRNA-297 and promotes cardiac repair. Biochem. Biophys. Res. Co. 523, 993-1000. doi: 10. 1016/j.bbrc.2019.12.116

Zhang, X. O., Wang, H. B., Zhang, Y., Lu, X. H., Chen, L. L., and Yang, L. (2014). Complementary sequence-mediated exon circularization. Cell 159, 134-147. doi: 10.1016/j.cell.2014.09.001

Zhang, Y., Zhang, X. O., Chen, T., Xiang, J. F., Yin, Q. F., Xing, Y. H., et al. (2013). Circular intronic long noncoding RNAs. Mol. Cell 51, 792-806. doi: 10.1016/j.molcel.2013.08.017

Zhao, Z. Z., Li, X. J., Gao, C. Y., Jian, D. D., Hao, P. Y., Rao, L. X., et al. (2017). Peripheral blood circular RNA hsa_circ_0124644 can be used as a diagnostic biomarker of coronary artery disease. Sci. Rep. 7:39918. doi: 10.1038/srep39918

Zheng, C., Niu, H., Li, M., Zhang, H., Yang, Z., Tian, L., et al. (2015). Cyclic RNA hsacirc000595 regulates apoptosis of aortic smooth muscle cells. Mol. Med. Rep. 12, 6656-6662. doi: 10.3892/mmr.2015.4264

Zheng, Q. P., Bao, C. Y., Guo, W. J., Li, S. Y., Chen, J., Chen, B., et al. (2016). Circular RNA profiling reveals an abundant circHIPK3 that regulates cell growth by sponging multiple miRNAs. Nat. Commun. 7:11215. doi: 10.1038/ ncomms 11215

Zhou, B., and Yu, J. W. (2017). A novel identified circular RNA, circRNA_010567, promotes myocardial fibrosis via suppressing miR-141 by targeting TGF-beta 1. Biochem. Biophys. Res. Commun. 487, 769-775. doi: 10.1016/j.bbrc.2017.04.044

Zhu, Y., Pan, W., Yang, T., Meng, X., Jiang, Z., Tao, L., et al. (2019). Upregulation of circular RNA CircNFIB attenuates cardiac fibrosis by sponging miR-433. Front. Genet. 10:564. doi: 10.3389/fgene.2019.00564

Zirkel, A., and Papantonis, A. (2018). Detecting circular RNAs by RNA fluorescence in situ hybridization. Methods Mol. Biol. 1724, 69-75. doi: 10.1007/ 978-1-4939-7562-4_6

Zou, M., Huang, C., Li, X., He, X., Chen, Y., Liao, W., et al. (2017). Circular RNA expression profile and potential function of hsa_circRNA_101238 in human thoracic aortic dissection. Oncotarget 8, 81825-81837. doi: 10.18632/ oncotarget. 18998

Conflict of Interest: The authors declare that the research was conducted in the absence of any commercial or financial relationships that could be construed as a potential conflict of interest.

Copyright (c) 2020 Zhang, Zhang, Wang, Zhao, Ding and Li. This is an open-access article distributed under the terms of the Creative Commons Attribution License (CC BY). The use, distribution or reproduction in other forums is permitted, provided the original author(s) and the copyright owner(s) are credited and that the original publication in this journal is cited, in accordance with accepted academic practice. No use, distribution or reproduction is permitted which does not comply with these terms. 